\title{
The effect of visual-vestibular conflict on the latency of steady-state visually induced subjective rotation
}

\author{
S. C. P. WONG and B. J. FROST \\ Queen's University, Kingston, Ontario K7L 3N6, Canada
}

\begin{abstract}
Two experiments were carried out to test the hypothesis that the long onset latency of steady-state subjective rotation induced by rotating a tall striped drum around a stationary observer is the result of visual-vestibular conflict. A reduction of this conflict should, therefore, reduce the length of the latency period. In Experiment 1, visual-vestibular conflict was reduced by providing the observers with corroborating vestibular stimulation at the start of optokinetic stimulation. Onset latencies were found to be significantly shortened with corroborating vestibular stimulation, but to be unaffected by noncorroborating vestibular stimulation. In Experiment 2, testing was done on a group of patients with unilateral (right labyrinth) Ménière's disease, which produces decreased vestibular sensitivity to rightward body rotation and increased vestibular sensitivity to leftward body rotation. Visual-vestibular conflict during subjective rotation to the right should therefore be decreased, while during leftward subjective rotation it should be increased. The prediction that the latency to steadystate subjective rotation should be shorter than normal with subjective rotation to the left was supported.
\end{abstract}

It has long been recognized that movement of an observer's entire visual environment leads to the illusory perception of bodily movement in the opposite direction, while the moving stimulus, paradoxically, appears to be stationary (Helmholtz, 1911; Mach, 1906; Thalman, 1921). Similar illusory selfmotion can be induced by a rotating sound field (Dodge, 1923; Lackner, 1977; Stein, 1910; Urbantschitsch, 1897) or even by kinesthetic rotary stimulation (Brandt, Buchele, \& Arnold, 1977; Lackner, 1977). Other forms of visually induced orientational changes, for example, tilt, pitch, and linear vertical and horizontal movements, can also be induced by visual stimulus motion that normally accompanies the corresponding movement of the observer in a rigid environment (Berthoz, Pavard, \& Young, 1977; Dichgans, Held, Young, \& Brandt, 1972; Johansson, 1977; Young, Oman, \& Dichgans, 1975).

The strong sensation of apparent orientational changes during visual stimulation has resulted in predictions of possible vestibular involvement in the illusion. This has been confirmed by a growing body

We wish to thank Stefan Michalski for expert technical assistance, D. M. L. Williams for screening appropriate patients, Kate Murphy and Georgette Craigie for typing the manuscript, and Jane Chown for proofreading. This research was supported by grants from the Natural Sciences and Engineering Research Council of Canada and the Medical Research Council of Canada to B. J. Frost. S. C. P. Wong's current address is: Department of Physiology, Faculty of Medicine, University of Saskatchewan, Saskatoon, Saskatchewan S7N 0W0, Canada. of neurophysiological literature showing strong visual-vestibular interactions during full-field visual stimulation (Allum, Graf, Dichgans, \& Schmidt, 1976; Dichgans \& Brandt, 1978; Dichgans, Schmidt, \& Graf, 1973; Gaunton \& Thomsen, 1976; Henn, Young, \& Finley, 1974; Waespe \& Henn, 1977). The vestibular system, though sensitive to acceleration, does not respond to constant velocity motion. Constant velocity motion of the body can be provided, to a large extent, by the visual system through the convergence of visual motion information onto vestibular centers.

Movement of the observer's entire visual field can be achieved by rotating a tall striped drum around an observer sitting in line with the axis of rotation. Rotation of the drum in one direction induces a compelling sensation of apparent subjective rotation in the opposite direction, while the rotating drum paradoxically appears to be stationary. Psychophysical studies of this effect (circularvection) show that observers, when exposed to the rotating drum, experience an initial period of apparent subjective acceleration together with the apparent deceleration of the rotating drum. This is followed by subjective rotation at a steady velocity which closely matches that of the rotating drum (Brandt, Dichgans, \& Koening, 1973; Wong \& Frost, 1978). Stimulation of the peripheral retinal areas produces a much stronger sensation of apparent movement than does stimulation of an equivalent area of central retina (Brandt et al., 1973). 
A recent study (Wong \& Frost, 1978) investigated, qualitatively and quantitatively, the developmental time course of subjective rotation at a constant velocity. Light onset illuminated the inside of the drum and signaled trial onset. Subjects typically experienced a 25- to 35-sec period of apparent subjective acceleration and a corresponding deceleration of the drum. This was followed by subjective rotation at a constant velocity and the almost complete stabilization of the rotating drum. However, the latencies to steady-state subjective rotation were independent of the drum velocity and remained at about $30 \mathrm{sec}$ with the three drum speeds used $(20,30$, and $40 \mathrm{deg} / \mathrm{sec})$.

The 25- to $30-\mathrm{sec}$ latency to reach a constantvelocity subjective rotation and drum stabilization is unlike everyday experience. When being artificially propelled, the observer immediately feels body movement in a rigid, stable environment. It was hypothesized (Wong \& Frost, 1978) that the long latency to constant-velocity subjective rotation could be due to the simultaneous presence of conflicting visual and vestibular inputs. During the initial exposure to an optokinetic stimulus moving at a constant velocity, the visual input would indicate subjective motion at a constant velocity whereas the vestibular input would indicate the absence of body movement. Both neurophysiological and psychophysical evidence support this hypothesis.

Neurophysiological experiments in which the firing rates of vestibular nuclei units in monkeys were monitored during optokinetic stimulation have shown that the firing rate increased gradually and reached a maximal rate after about $30 \mathrm{sec}$ of stimulation (Waespe \& Henn, 1977). However, when both optokinetic and vestibular stimulations were applied together (i.e., by physical rotation of the monkey inside a lighted drum), the latency to a steady-state discharge rate was reached in about $10 \mathrm{sec}$.

Dichgans et al. $(1973,1974)$ and Young et al. (1975) have shown that subjective tilt, pitch, and roll can be induced by visual stimulation alone. However, the magnitude of these visually induced orientation changes was limited. Dichgans et al. and Young et al. suggested that the small magnitude of these changes could be due to the simultaneous presence of conflicting orientation information from the graviceptive receptors and the visual system. While visual motion information might indicate the presence of subjective tilt, pitch, and roll, the graviceptive input would indicate the absence of such changes. Their hypothesis was supported by the observation that when the graviceptors were made less sensitive by changes in head position, thereby reducing the visualvestibular conflict, the magnitude of the visually induced orientation changes showed a significant increase.
Two experiments were designed to test the hypothesis that a reduction in visual-vestibular conflict would reduce the latency to the onset of steadystate subjective rotation induced by a large rotating striped drum.

\section{EXPERIMENT 1}

Subjective rotation was induced, as reported earlier (Wong \& Frost, 1978), by rotating a tall striped drum around an observer sitting in line with the axis of rotation. Visual-vestibular conflict that could have been occurring at the initiation of optokinetic stimulation was reduced by providing the observer with corroborating vestibular stimulation. The vestibular stimulation was in the form of a brief period of rotary acceleration of the observer's chair.

\section{Method}

Subjects. Eight subjects, four male and four female unpaid university students between the ages of 19 and 24 , were tested. All had normal or corrected vision and no previous complaints of vestibular problems.

Procedure. The subject sat inside a drum, $2 \mathrm{~m}$ tall $\times 1.5 \mathrm{~m}$ in diameter, the inside wall of which was lined with alternating vertical black and white stripes, each $4 \mathrm{deg}$ in width, the Michelson contrast ratio of the stripes was $.81[(\mathrm{~L} \max -\mathrm{L} \min ) /(\mathrm{L} \max +$ $L$ min)]. The drum could be rotated in a clockwise or counterclockwise direction at speeds that could be varied from a few $\mathrm{deg} / \mathrm{sec}$ to about $180 \mathrm{deg} / \mathrm{sec}$. The subject sat on a chair which could also be partially rotated smoothly through about $60 \mathrm{deg}$ clockwise or counterclockwise from a central position. A footrest attached to the chair prevented the subject from orienting himself through contact with the floor. Circular microtorque potentiometers were connected to the axle of the drum and the chair. The velocity and the direction of both chair and drum rotation could thus be monitored independently and continuously throughout the experiment. The voltage variations produced by the rotation of both potentiometers were recorded on separate channels of a Beckman polygraph.

The partial rotation of the chair was made possible by a compressed spring-and-lever system installed adjacent to the footrest of the chair. A hinged bar compressed a large spring, which was held in the compressed position by a powerful electromagnet. When the hinged bar was in the compressed position, the chair could be rotated such that the footrest, to which it was attached, was in contact with the bar. Deactivation of the electromagnet caused the hinged bar to move, due to the decompression of the spring. The movement of the bar pushed the footrest, and therefore the rest of the chair and the observer sitting on it, through an angle of about $30 \mathrm{deg}$. The electromagnet was then remagnetized by a self-resetting timer and, before the next trial, the hinged bar was moved by hand back to the resting position and the chair was repositioned so that the footrest was again in contact with the hinged bar. The lever system was set up to propel the chair in clockwise directions only.

Thus seated, with his head supported by a headrest, the subject's entire visual field was filled by the wall of the drum. Even illumination inside the drum was provided by an incandescent light source located above the subject's head. Extraneous noise was masked by white noise delivered through headphones the subject wore throughout the experiment.

The subject sat in the dark inside the drum, which was then set into clockwise or counterclockwise rotation. After the drum had reached a constant velocity of $30 \mathrm{deg} / \mathrm{sec}$, the inside light 
was turned on to signal the beginning of the trial. On half of the trials, light onset was coupled with the demagnetization of the electromagnet and thus produced partial chair rotation.

The rotation of the chair consisted of a brief period of very rapid acceleration (mean acceleration, $239.88 \mathrm{deg} / \mathrm{sec}^{2}, \mathrm{SE}=$ 21.64) which lasted for a mean of $.17 \mathrm{sec}(\mathrm{SE}=.02)$. This was followed by a longer period (mean duration, $.90 \mathrm{sec}, \mathrm{SE}=.13 \mathrm{sec}$ ) of slower deceleration (mean, $29.40 \mathrm{deg} / \mathrm{sec}^{2}, \mathrm{SE}=6.69$ ). The magnitude of the arc of chair rotation was about $30 \mathrm{deg}$ (mean, $30.26 \mathrm{deg}, \mathrm{SE}=4.12 \mathrm{deg}$ ) and lasted for a mean of $1.1 \mathrm{sec}$ $(\mathrm{SE}=.15)$.

Each subject was tested in each of the following four conditions: Condition A-counterclockwise drum rotation (clockwise apparent subjective rotation) with no chair rotation; Condition Bcounterclockwise drum rotation (clockwise apparent subjective rotation) with clockwise chair rotation; Condition $\mathrm{C}$-clockwise drum rotation (counterclockwise apparent subjective rotation) with no chair rotation; Condition D-clockwise drum rotation (counterclockwise apparent subjective rotation) with clockwise chair rotation.

The subjects were instructed to give magnitude estimates (Stevens, 1957) of the apparent speed of drum and subjective rotations at 5 -sec intervals throughout the 1 -min trial. Five seconds after light onset and every $5 \mathrm{sec}$ thereafter, a tone was sounded, immediately after which the subject was to give the two estimates. Shortly before the trial began, the rotating drum $(30 \mathrm{deg} / \mathrm{sec})$ was illuminated briefly $(1.5 \mathrm{sec})$ and the subject was told to use that as the standard speed and to assign to it the number 10 . When the standard was presented, the exposure of the subject to the moving drum was too short to produce subjective rotation. Thus, the standard speed, represented by the number 10 , should have been close to the physical speed of the drum (see Wong \& Frost, 1978).

Each subject was given two trials in each of the four conditions, and the sequence in which the trials were given was counterbalanced between subjects.

\section{Results}

The results of the first experiment are shown in Figures 1-4. In Figure 1, the subjects' mean magnitude estimates of the velocity of drum rotation and the velocity of subjective rotation were plotted for Conditions A and B. In Figure 3, mean magnitude estimates of the velocity of drum rotation and subjective rotation were plotted for Conditions $\mathrm{C}$ and $\mathrm{D}$.

In Figure 1, the pattern of results obtained for drum and subjective rotations in the absence of chair rotation were very similar to that reported earlier (Wong \& Frost, 1978). After the trial began, the velocity of subjective rotation increased rapidly, reaching a steady level about $30 \mathrm{sec}$ after light onset. Similarly, the velocity of drum rotation decreased, reaching a steady level at about the same time.

However, when light onset was coupled with chair rotation in the same direction as the expected direction of subjective rotation (Condition B), the velocity of subjective rotation increased much more rapidly, reaching an asymptote about $15 \mathrm{sec}$ after light onset. Similarly, the apparent velocity of drum rotation decreased more rapidly, reaching a steady level about $20 \mathrm{sec}$ after light onset. Within-subject $2 \times 6$ analyses of variance showed that over the first $30 \mathrm{sec}$ of the trial, Condition A was significantly different from Condition $B$ for both the magnitude $\triangle \mathrm{SR}$ and same direction CR

- SR alone

- DR and same direction CR

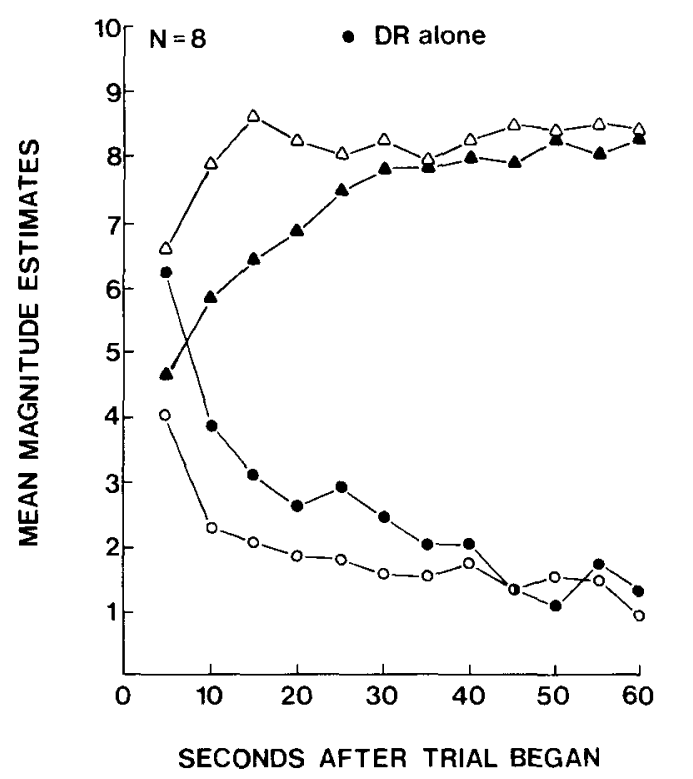

Figure 1. Mean magnitude estimates of subjective rotation (SR) and drum rotation (DR) speeds with and without same-direction chair rotation (CR).

$\triangle$ SR and same direction CR

- SR alone

○ DR and same direction CR

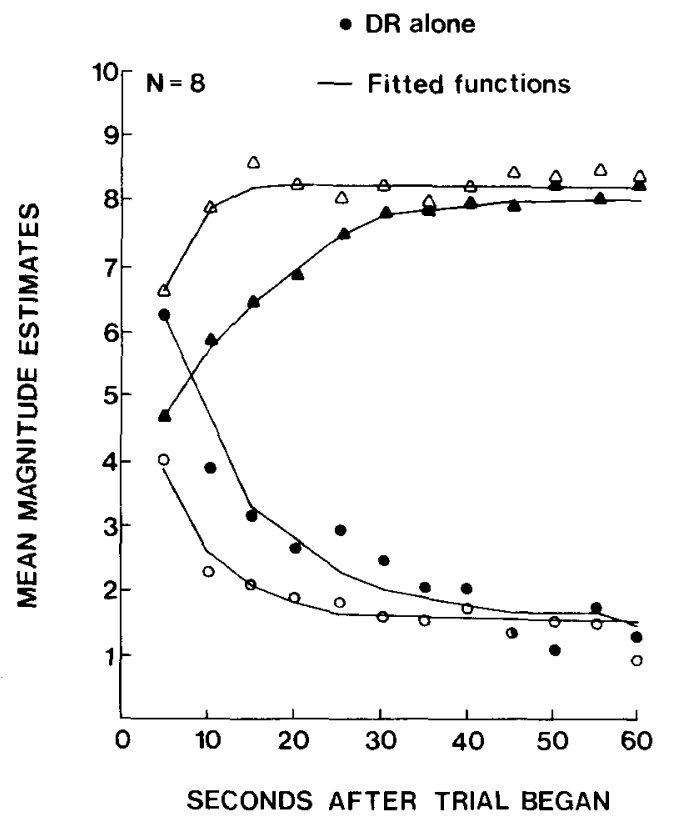

Figure 2. Obtained values and fitted functions for the mean magnitude estimates of subjective rotation (SR) and drum rotation (DR) speeds with and without same-direction chair rotation (CR). 
of drum rotation and subjective rotation $[\mathrm{Fs}(1,84)=$ 9.2 and 13.9, respectively; in each case, $\mathrm{p}<.01]$. However, there was no difference between Condition $\mathrm{A}$ and Condition $\mathrm{B}$ from the 35- to the $60-\mathrm{sec}$ mark for both drum and subjective rotation $[\mathrm{Fs}(1,84)=$ .6 and .1 , respectively; $\mathrm{p}>.1$, in each case].

Between Condition $C$ and Condition D, no significant difference was found for the apparent velocity of drum rotation or subjective rotation either in the first $30 \mathrm{sec}[\mathrm{Fs}(1,84)=.3$ and .1 , respectively; $\mathrm{p}>.1$, in each case] or in the subsequent $30 \mathrm{sec}$ of stimulation $[\mathrm{Fs}(1,84)=2.4$ and 1.3 , respectively; $\mathrm{p}>.1$, in each case]. Chair rotation opposite to the direction of expected subjective rotation does not seem to have an effect on the onset latency of either subjective rotation or drum rotation.

In order to obtain a more objective description of the drum and subjective rotational changes over the trial and to derive a quantitative estimate of the asymptote of steady-state subjective rotation, curves were fitted to the data (see Appendix A).

The obtained data and the calculated best-fitting exponential function $\left(y=k+b e^{-a x}\right)$ are shown in Figures 2 and 4.

The correlation coefficients for the obtained and the calculated functions for both drum and subjective rotation are .99 and .99 , respectively. Thus, the obtained data can be described very well by the exponential function.

It is quite apparent, by visual inspection alone, that the asymptotes for both drum and subjective rotation for Condition $\mathrm{A}$ were reached about $30 \mathrm{sec}$ after stimulation began. However, it is important, for the present argument, to have an objective indication of when, during the 1-min trial, asymptote was first reached. The fitted exponential functions, which so closely approximated the obtained values, were used to estimate this point, as outlined in Appendix $B$.

Such computation yielded, for Condition A, asymptotic values for subjective and drum rotations at the $35-$ and $30-$ sec points, respectively. These values are very similar to the ones obtained by visual inspection. Using the same method of computation, the product-moment correlation coefficient between the obtained and the predicted magnitude estimates functions for different combinations of drum and chair rotations and the corresponding asymptotic points were calculated and are shown in Table 1.

A comparison between the asymptotic points for Conditions A and B shows that the latencies to steadystate subjective rotation and drum rotation were substantially shorter when chair rotation was in the same direction as subjective rotation. However, the asymptotic values for Conditions $\mathrm{C}$ and $\mathrm{D}$ indicate that onset latencies for drum and subjective rotation were not affected when the direction of chair rotation was opposite to that of subjective rotation.

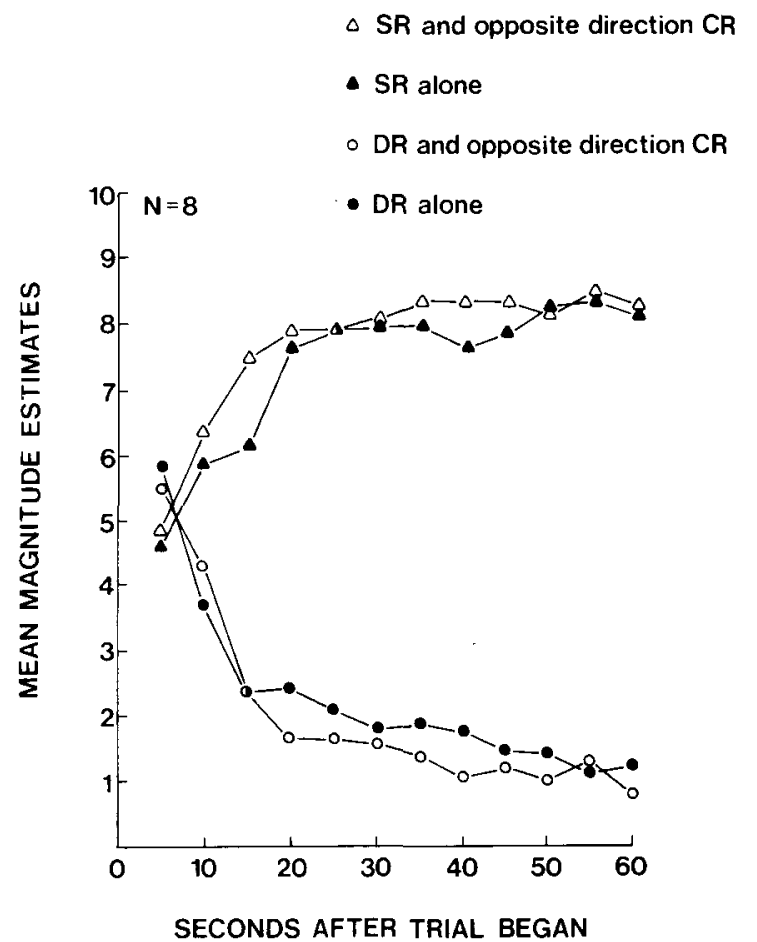

Figure 3. Mean magnitude estimates of subjective rotation (SR) and drum rotation (DR) speeds with and without oppositedirection chair rotation (CR).

$\triangle$ SR and opposite direction CR

- SR alone

- DR and opposite direction CR

- DR alone

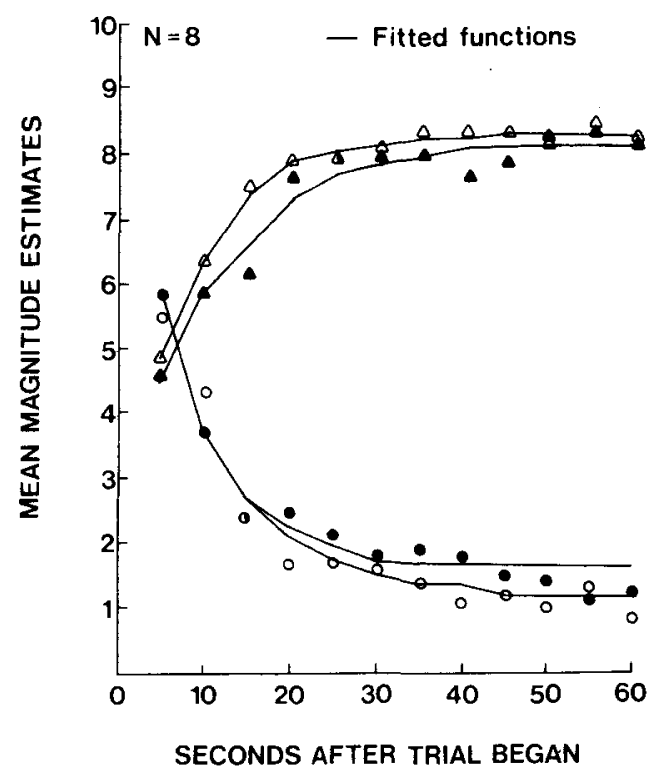

Figure 4. Obtained values and fitted functions for the mean magnitude estimates of subjective rotation (SR) and drum rotation (DR) speeds with and without opposite-direction chair rotation (CR). 


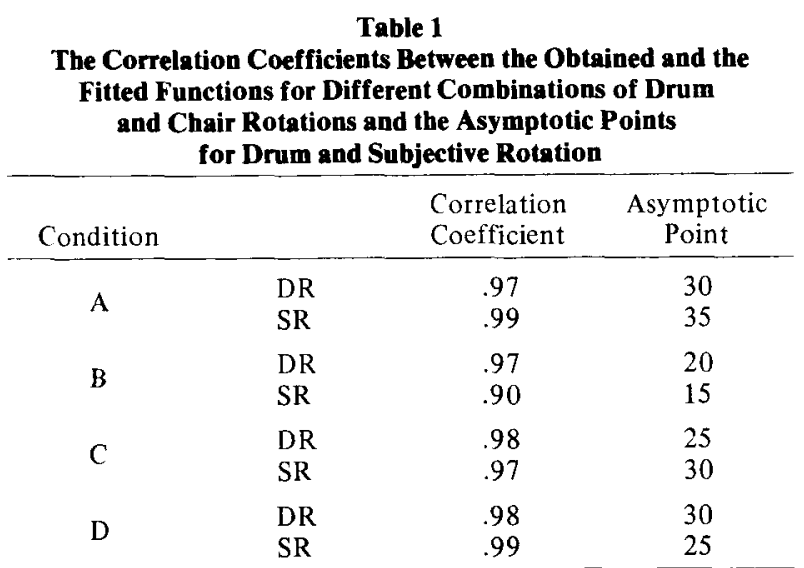

Note-Asymptotic points are given in seconds. $S R=$ subjective rotation DR $=$ drum rotation.

\section{Discussion}

The first experiment demonstrated the results of presenting observers with corroborating and noncorroborating vestibular stimuli at the initiation of optokinetic stimulation. In the presence of corroborating signals (Condition $\mathrm{B}$ ), the latencies to reach both a steady-state subjective rotation and stabilization of the visual field were significantly shorter than when signals from the vestibular system indicating body movement were absent (Condition A). However, with noncorroborating visual and vestibular signals generated by rotating the chair opposite to the direction of expected subjective rotation (Condition $\mathrm{C}$ ), the latency measurements for both subjective rotation and drum stabilization were not significantly different from the condition in which no chair rotation occurred (Condition D). These results support the hypothesis that the rather long latency to steady-state subjective rotation results from the presence of conflicting visualvestibular signals, indicating self-movement. When the conflict was reduced, as in Condition B, the latency was shortened significantly.

A comparison between Conditions $C$ and D showed that there was no difference in the onset latencies of steady-state subjective rotation or drum stabilization for the two conditions. This implies that the magnitude of visual-vestibular conflict is similar whether the observer is maintained in a stationary position or is being propelled in the opposite direction. This is not unexpected. The vestibular system typically maintains a steady discharge rate in the resting state. Physical acceleration or optokinetic stimulation modulates the discharge rate in either direction (Goldberg \& Fernandez, 1971; Waespe \& Henn, 1977). The vestibular system can therefore signal both the presence and the absence of acceleration. It follows that vestibular signals indicating no acceleration or acceleration opposite to the direction of expected subjec- tive rotation can be considered to be equivalent. They are equivalent in the sense that both are in conflict with the optokinetic stimulation. Both Condition $\mathrm{C}$ and Condition $\mathrm{D}$ would then result, as hypothesized, in the prolonged latency to steady-state subjective rotation.

In summary, findings from the present experiment support the hypothesis that visual-vestibular conflict is the cause of the long latency to steadystate subjective rotations. A reduction of the conflict through corroborating vestibular stimulation produced a significantly shorter latency period.

\section{EXPERIMENT 2}

If the visual-vestibular conflict is reduced through the reduction of vestibular sensitivity, there should also be a corresponding shortening of the onset latency of steady-state subjective rotation. Some recent evidence supports this hypothesis. Hokendorf, Bles, and Brandt (1977) have shown that a direction-specific reduction in latency of subjective rotation occurs in patients with a vestibular tonus imbalance due to an acute unilateral labyrinthine lesion. The lesion could have lowered the sensitivity of the vestibular system, thereby reducing the conflict between the visual and the vestibular systems and consequently decreasing the onset latency of subjective rotation.

When the horizontal vestibular canal system on one side is considered independently of the contralateral system, its sensitivity to clockwise and counterclockwise rotations differs. Ewald and others (Ewald, 1892, cited in Howard \& Templeton, 1966; Groen, 1960) have shown that sensitivity of the horizontal canal to rotation is higher when the cupula is deflected in an utriculopetal rather than utriculofugal direction (Ewald's second law). An utriculopetal cupula deflection is produced when the head is rotated ipsilateral to the side under consideration. In other words, the horizontal canal on the left side is more sensitive to horizontal head rotation to the left (ipsilateral rotation) than it is to horizontal head rotation to the right (contralateral rotation). The opposite is true for the horizontal canal on the right side. For people with normal vestibular function on both sides, no net advantage in terms of sensitivity to horizontal head rotation to either side should be evident. The higher sensitivity of the left horizontal canal to leftward head rotation is balanced by the higher sensitivity of the right horizontal canal to rightward head rotation. However, for those having a depressed or a complete absence of horizontal canal responsiveness on one side, they should show a normal sensitivity to horizontal head rotations ipsilateral to the unaffected side and a lowered sensitivity to horizontal head rotation ipsilateral to the affected side. The difference in sen- 
sitivities to the two directions of horizontal head or body rotation should produce, according to our hypothesis, a difference in the onset latency of steady-state subjective rotations induced by optokinetic stimulation.

The onset latency of subjective rotation towards the side with normal sensitivity should be comparable to that of a normal group. On the other hand, onset latency of subjective rotation towards the less sensitive side should be lower than the normal value. These predictions are based on the assumption that the magnitude of visual-vestibular conflict is reduced during the induction of subjective rotation towards the less sensitive side but remains the same when subjective rotation is induced towards the normal side.

An opportunity to test this hypothesis was made possible because of the existence of a vestibular disorder, called Ménière's disease, which can affect one or both vestibular systems.

Ménière's disease is a type of peripheral labyrinthine disorder which can lead to the gradual reduction of vestibular sensitivity in one or both vestibular systems (Paparella \& Shumrick, 1976). Ménière's appears to affect only the labyrinth, with no central neural involvement. For $80 \%$ to $90 \%$ of those affected, the disease is limited to the labyrinth on one side. Quite often both the cochlea and the vestibular systems are affected. During an acute attack, the patients usually complain of having whirling sensations accompanied by pallor, nausea, vomiting, and prostration. As the disease progresses, there is a progressive destruction of vestibular hair cells, and this leads to a gradual reduction of vestibular sensitivity. The etiology of the disease is essentially unknown.

The degree of reduction in vestibular sensitivity in the individual vestibular system can be measured by the caloric test, which involves irrigating the external auditory meatus with warm $\left(44^{\circ} \mathrm{C}\right)$ or cold $\left(30^{\circ} \mathrm{C}\right)$ water while the head is tilted back $60 \mathrm{deg}$ to bring the horizontal semicircular canal into the vertical plane. Typically, after a caloric test, the velocities of a number of the fastest slow-phase eye movements are measured on the eye-movement record and their mean computed. The mean velocities of the slow-phase eye movements generated by caloric irrigation to the left and the right auditory meatuses are then compared. A left-right velocity difference that is more than $20 \%$ of the sum of leftright velocities is considered to be an indication of depressed vestibular responsiveness at the side with the lower slow-phase velocity.

For the second experiment, a group of five subjects who had had Ménière's disease only in the right labyrinth were tested. Their vestibular sensitivities were measured in both ears by the caloric test and were found to be below normal by $36 \%$ to
$100 \%$ in the affected side but completely normal in the unaffected side. More detailed information on this group of subjects is given in the subject section.

\section{Method}

Subjects. The patient subjects were one male and four female adults. All of them were diagnosed as having had Ménière's disease. None of the subjects was undergoing treatment at the time of testing. Their personal data and a brief account of their diagnosis is presented in Table 2.

Care was taken to select a group of subjects with only unilateral Ménière's disease and no additional vestibular or other medical complications. The strictness of the selection criteria and the unavailability of some subjects due to personal and other reasons, restricted both the number and the type of subjects available for testing. Ideally, a second group of subjects with Ménière's disease only in the left vestibular system should also have been tested, but not enough patients meeting these criteria were available. The clinical diagnosis and the caloric testing of the subjects were done under the supervision of D. M. L. Williams, Head of the Department of Otolaryngology at the Kingston General Hospital, Kingston, Ontario.

The normal group consisted of three university students (one male, two female) between the ages of 20 and 27 who had no previous history of vestibular complaints.

All subjects had normal or corrected-to-normal vision.

Procedure. The apparatus was identical to that used in Experiment 1 , except that no chair rotation was used in the present experiment.

The subjects sat in the dark inside the drum, which was set into clockwise or counterclockwise rotation. After the drum reached a constant velocity, the inside light was turned on to signal the beginning of a trial. Light offset indicated the end of a trial. No fixation point was provided in this experiment. With the drum rotating at $30 \mathrm{deg} / \mathrm{sec}$, the subjects were required to press one of three buttons throughout the 1-min trial to indicate whether they observed only the drum rotating, a combination of drum rotation and subjective rotation, or subjective rotation alone. The duration and the temporal location of each press was recorded on a Beckman polygraph (see Wong \& Frost, 1978). Each subject was given two clockwise and two counterclockwise trials.

The magnitude estimation technique, which requires the reporting of drum and subjective rotation speeds every $5 \mathrm{sec}$ throughout the 1-min trial, was not used in the present experiment. The magnitude estimation technique, it was feared, might have been too demanding for the patient subjects, some of whom were from out of town locations and could be present for only a single short testing session.

\section{Results}

Figure 5 shows the percentage of trials in which subjects reported only subjective rotation (with no

Table 2

Vestibular Responsiveness, as Determined by Caloric Test, of Subjects With Ménière's Disease (Experiment 2)

\begin{tabular}{ccc}
\hline Subject & Age & Right Ear \\
\hline I.B. & 47 & 54 \\
C.G. & 62 & 100 \\
N.G. & 54 & 69 \\
M.H. & 51 & 60 \\
T.S. & $?$ & 36 \\
\hline
\end{tabular}

Note-Age is given in years. Entries for the right ear indicate percentage below normal; the left ear was normal in each case. 


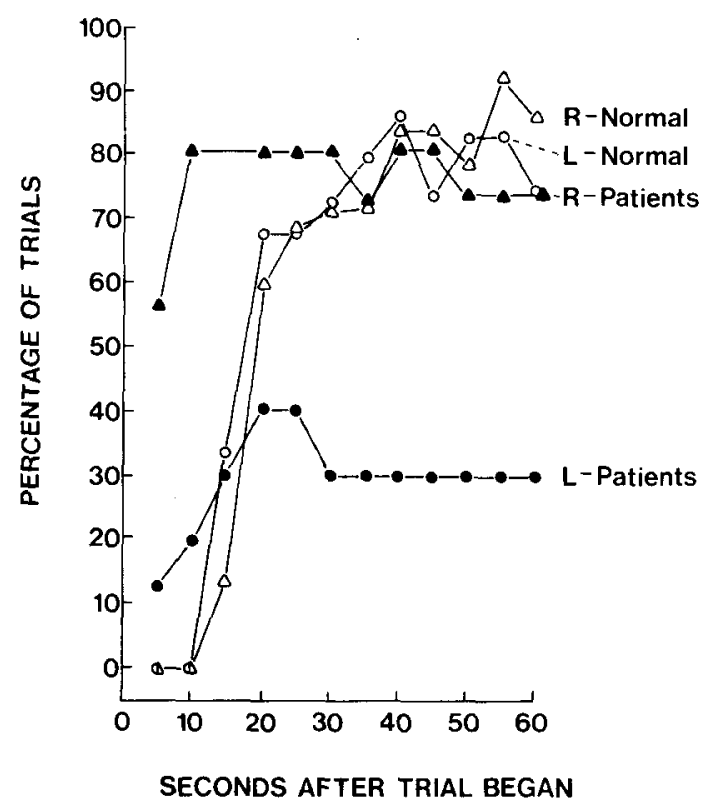

$R, L$ : Subjective rotation to the right $(R)$ or the left $(L)$

Figure 5. Reports of subjective motion by a group of subjects with right unilateral Ménière's disease and a group of normals over a 1-min trial.

perceived drum rotation) as a function of both time from the beginning of a trial and the direction of drum rotation. For the normal group, the pattern of results is very similar to the observations reported previously (Wong \& Frost, 1978).

Subjective rotation reached a steady state about $35 \mathrm{sec}$ after light onset. When the curve-fitting procedures used in Experiment 1 were applied to the present results for the normal group, the function $y=k+b^{-a x}$ was again found to fit the data well. The correlation between the obtained and the fitted function was .88 . However, since the dependent variable in this experiment was not in the form of a magnitude estimate, the procedures used to obtain the asymptotic time cannot be applied here. Instead, a nonparametric method was used to analyze the data.

Using a Kruskal-Wallis one-way analysis of variance by ranks (Kirk, 1968) in this and in the following comparisons, it was found that there was no difference in the results for the two directions of drum rotation for the control group either during the first $30 \mathrm{sec}$ or in the subsequent $30 \mathrm{sec}$ of the trial $(\mathrm{H}=.1, \mathrm{p}>.1$, and $\mathrm{H}=1.4, \mathrm{p}>.1$, respectively). Therefore, in the following analysis, the results of the normal group during clockwise and counterclockwise drum rotation are combined.

With the patient group, an entirely different pattern of results emerged. When the drum was rotated to the subject's left, producing subjective rotation to the subject's right, a steady level of subjective rotation was reached in $10 \mathrm{sec}$ or less after light onset. However, when the drum was rotated to the subject's right, producing subjective rotation to the subject's left, a steady state comparable to that reached during leftward rotation was not reached within the 1-min testing period. For the patient group, significant differences occur between the two directions of subjective rotation in the first $30 \mathrm{sec}$ and also in the subsequent $30 \mathrm{sec}$ of the trial $(H=8.3, p<.01$, in both cases).

When the results of the patient group during subjective rotation to the right were compared with those of the normals, significant differences were obtained during the first $30 \mathrm{sec}(\mathrm{H}=5.0, \mathrm{p}<.05)$ but not during the last $30 \mathrm{sec}$ of the trial $(\mathrm{H}=3.7$, $\mathrm{p}>.05$ ).

However, during subjective rotation to the left, the results of the patients were not significantly different from those of the normals in the first $30 \mathrm{sec}$ $(\mathrm{H}=.1, \mathrm{p}>.1)$. A significant difference was obtained during the subsequent $30 \mathrm{sec}$ of the trial $(\mathrm{H}=8.3, \mathrm{p}<.01)$.

When the curve-fitting procedures were applied to the present data for the patient groups, neither function gave a good fit to data obtained when the patients experienced subjective rotation to the right. A good fit was defined arbitrarily as a correlation of .5 or more between the obtained and the fitted functions. This is not unexpected, since the function is relatively flat except during the initial $5 \mathrm{sec}$ of stimulation. However, data obtained when patients experienced subjective rotation to the left could be fitted again by the function $y=k+b e^{-a x}$.

The correlation between the obtained and the fitted function was $\mathbf{. 7 5}$.

\section{Discussion}

The results provide additional support for the hypothesis that the onset latency of steady-state subjective rotation is affected by the magnitude of visual-vestibular conflict that exists during optokinetic stimulation. A reduction in sensitivity to the right vestibular system in the present patients produced an onset latency to steady-state subjective rotation that was much shorter when the rotation was to the right than when it was to the left. It should be noted that a level of subjective rotation to the left (induced by drum rotation to the right) comparable to that of the normal subjects was not reached by the patients during the 1-min testing period. A longer duration of testing, to see if patients do eventually reach an asymptote similar to that of the normals, was not attempted. However, noting the stability of the scores in the last $30 \mathrm{sec}$ of testing, it seemed unlikely that the patients would reach a higher level even if the testing had been prolonged. The latter re- 
sult was not predicted by the hypothesis. However, such a pattern of results would be obtained if it were assumed that the two vestibular systems do not operate independently of each other. There is some neurophysiological evidence to support this assumption. Shimazu and Precht (1966) have shown that there are strong inhibitory influences on Type I vestibular neurons originating from the contralateral labyrinth. Type I neurons depolarize and hyperpolarize to ipsi- and contralateral rotation, respectively, and are considered to be major vestibular neurons because of their lower threshold to rotary stimulation (Precht, Shimazu, \& Markham, 1966). Weak electrical stimulation of the contralateral vestibular nerve inhibits the spontaneous discharges of Type I neurons, whereas midline incisions which interrupt the commissural fibers from the contralateral labyrinth completely abolish such inhibition. These and other related experiments have led Shimazu and Precht (1966) to conclude that the increase in discharge rate of Type I vestibular neurons during ipsilateral rotation is in part the result of the withdrawal of contralateral labyrinthine inhibition. It could be argued that after unilateral labyrinthine lesion, the Type I neurons on the intact side could exhibit a degree of hypersensitivity to ipsilateral rotary stimulation as a result of the removal of contralateral inhibition. A certain degree of hypersensitivity could still persist even after central compensation.

Type II neurons, which increase their discharge frequency during horizontal angular acceleration toward the contralateral side and decrease their discharge frequency to ipsilateral rotation, have been shown to be influenced mainly by the contralateral horizontal canal (Shimazu \& Precht, 1966). They also respond even if the ipsilateral labyrinth has been removed, but are silent after cutting the commissural fibers. Removal of the contralateral labyrinthine input therefore would eliminate the activity of the Type II neurons on the intact side.

Taken together, these findings would suggest that, with unilateral vestibular lesions, sensitivity to rotation ipsilateral to the intact side should increase whereas sensitivity to rotation contralateral to the intact side should decrease. This would lead to a higher than normal level of visual-vestibular conflict during rotation ipsilateral to the intact side and a lower than normal level of visual-vestibular conflict during subjective rotation contralateral to the intact side. According to this argument, the former would lead to a longer and the latter to a shorter than normal onset latency of visually induced subjective rotation. Results in support of this position were obtained in the second experiment.

The results from the present experiment, when interpreted in the light of the more recent electro- physiological findings which suggest significant intervestibular excitatory and inhibitory interactions, confirm the hypothesis (Wong \& Frost, 1978; Dichgans \& Brandt, 1978) that onset latency of subjective rotation is probably a function of the visualvestibular conflict.

\section{REFERENCES}

Allum, J. H. J., Graf, W., Dichgans, J., \& Schmidt, C. L. Visual vestibular interactions in the vestibular nuclei of the goldfish. Experimental Brain Research, 1976, 26, 463-485.

Berthoz, A., Pavard, B., \& Young, L. R. Perception of linear horizontal self-motion induced by peripheral vision (linearvection). Experimental Brain Research, 1975, 23, 471-489.

BrandT, Th., Buchele, W., \& Arnold, F. Arthrokinetic nystagmus and ego-motion sensation. Experimental Brain Research, 1977, 30, 331-338.

Brandt, Th., Dichgans, J., \& Koenig, E. Differential effects of central versus peripheral vision on egocentric and exocentric motion perception. Experimental Brain Research, 1973, 16, 476-491.

Dichgans, J., \& Brandt, Th. Visual-vestibular interaction and motion perception. In J. Dichgans \& E. Bizzi (Eds.), Cerebral control of eye movements and motion perception. BaselNew York: Karger, 1972.

Dichgans, J., \& Brandt, Th. Visual-vestibular interaction: Effects on self-motion perception and postural control. In R. Held, H. Leibowitz, \& T. L. Teuber (Eds.), Handbook of sensory physiology (Vol. 8) Perception. Berlin: Springer, 1978.

Dichgans, J., Diener, H. C., \& Brandt, Th. Optokinetic graviceptive interaction in different head positions. Acta Otolaryngologica, 1974, 78, 391-398.

Dichgans, J., Held, R., Young, L., \& Brandt, Th. Moving visual scenes influence the apparent direction of gravity. Science, 1972, 178, 1217-1219.

Dichgans, J., Schmidt, C. L., \& Graf, W. Visual input improves the speedometer function of the vestibular nuclei in the goldfish. Experimental Brain Research, 1973, 18, 319-322.

DoDGE, R. Thresholds of rotation. Journal of Experimental Psychology, 1923, 6, 471-489.

Ewald, J. R. Physiologische Untersuchungen über das Endorgan des NOctavus. Wiesbaden: Bergmann, 1892.

Gaunton, N. G., \& Thomsen, D. Otolith-visual interactions in single units of cat vestibular nuclei. Neuroscience Abstracts, 1976, 2, 1526.

Goldberg, J. M., \& Fernandez, C. Physiology of peripheral neurons innervating semicircular canals of the squirrel monkey. I. Resting discharge and response to constant angular acceleration. Journal of Neurophysiology, 1971, 34, 635-660.

Groen, J. J. On the repeal of Ewald's second law. Acta Otolaryngology (supplement), 1960, 159, 42-46.

Helmholtz, H. von. Helmholtz's treatise on physiological optics (J. P. C. Southall, Ed. and trans.). New York: Dover, 1962. (Originally published 1909-1911.)

Henn, V., Young, L. R., \& Finley, C. Vestibular nucleus units in alert monkeys are also influenced by moving visual fields. Brain Research, 1974, 71, 144-149.

Hokendorf, H., Bles, W., \& Brandt, Th. Direction specific reduction of circularvection latencies as a measure of latent labyrinthine vertigo. Pflügers Archiv Supplement, 1977, 368, R45, 179

Howard, L. R., \& Templeton, W. B. Human spatial orientation. London: Wiley, 1966.

Johansson, G. Studies on visual perception of locomotion. Perception, 1977, 6, 365-376.

Kır, R. E. Experimental design: Procedures for behavior sciences. Belmont, Calif: Brooks/Cole, 1968. 
LACKNE R, J. R. Induction of illusory self-rotation and nystagmus by a rotating sound-field. Aviation, Space and Environmental Medicine, 1977, 48(2), 129-131.

Масн, E. The analysis of sensation (5th ed., S. Waterlow, trans.). New York: Dover, 1959. (Originally published, 1866; 5 th ed. published, 1906.)

Paparella, M. M., \& Shumrick, D. A. (Eds.) Scientific foundations of otolaryngology. Philadelphia: Heinemann, 1976.

Precht, W., Shimazu, H., \& Markham, Ch. H. A mechanism of central compensation of vestibular function following hemilabyrinthectomy. Journal of Neurophysiology, 1966, 29, 996-1010.

Shimazu, H., \& Precht, W. Inhibition of central vestibular neurons from the contralateral labyrinth and its mediating pathway. Journal of Neurophysiology, 1966, 29, 462-492.

Ste in, S. von. Schwindel (Audiokinesis externa et interna). Leipzig: Lessier, 1910.

Thalman, W. A. The aftereffect of seen movement when the whole visual field is filled by a moving stimulus. American Journal of Psychology, 1921, 32, 429-441.

Urbantschitsch, V. Über Storungen des Fleichgewichtes und Scheinbewegungen. Zeitschrift für Chrenheilkunde, 1897, 31, 234-294.

Waespe, W., \& Henn, V. Neuronal activity in the vestibular nuclei of the alert monkey during vestibular and optokinetic stimulation. Experimental Brain Research, 1977, 27, 523-538.

Wong, S. C. P., \& Frost, B. J. Subjective motion and acceleration induced by the movements of the observer's entire visual field. Perception \& Psychophysics, 1978, 28, 115-120.

Young, L. R., Oman, C. M., \& Dichgans, J. M. Influence of head orientation on visually induced pitch and role sensation. Aviation, Space and Environmental Medicine, 1975, 46(3), 264-268.

\section{APPENDIX A}

Two functions were fitted to the obtained data by the least squares method. The functions were an exponential function, $y=k+b e^{-a x}$, and a hyperbolic function, $y=k+b x^{-1}$. They were chosen because both of them satisfied the requirement that as $x$ approaches infinity, $y$ approaches $k$, where $x=$ time after trial began, $y=$ magnitude estimates, $k=$ asymptotic value, and $a, b=$ constants.

The value of $b$ is negative when $y$ increases as $x$ increases, that is, during magnitude estimation of subjective rotation. The value of $b$ is positive when y decreases as $\mathrm{x}$ increases, that is, during magnitude estimation of drum rotation. The better fitting function is the one with the higher product moment correlation computed between the obtained and the fitted data. It was found that, for the present experiment and also for the data reported earlier (Wong \& Frost, 1978), the exponential function gave a better overall fit.

\section{APPENDIX B}

The values of $y_{s}$ in the function $y_{s}=k+b e^{-a x}$ for subjective rotation increases as $\mathrm{x}$ increases and approaches $a$ as $x$ approaches infinity. When the increment of $y_{s}$ is insignificantly small, the value of $\mathrm{x}$ is the point in time when asymptote is reached.

The value $y_{s}$ is the magnitude estimate of the speeds of subjective rotation. The increment of y over time is therefore subjective acceleration. The point $\mathrm{x}$ when the increment of $y$ is insignificant is the threshold of subjective acceleration, or the point on the asymptote.

The thresholds of body acceleration have been determined empirically by a number of methods. Their values vary between .12 and $2 \mathrm{deg} / \mathrm{sec}^{2}$, depending on the method used for their determination (Howard \& Templeton, 1966). A value of $.10 \mathrm{deg} / \mathrm{sec}^{2}$ was chosen for the present calculation to ensure that acceleration was most likely at the point that would be considered the threshold of acceleration.

The change in magnitude estimate that corresponds to the threshold of acceleration has to be calculated as follows. Subjects in the present experiment were asked to use the drum speed of $30 \mathrm{deg} / \mathrm{sec}$ as the standard and to assign to it a value of 10 . A change in the perceived speed of $30 \mathrm{deg} / \mathrm{sec}$ would correspond to a change in the size of the magnitude estimate by 10 . Hence, a change in the perceived speed, that is, an acceleration of $.10 \mathrm{deg} / \mathrm{sec}^{2}$, would correspond to a change in the magnitude estimate by .1 $(10 / 30)$ per sec, or $.0333 / \mathrm{sec}$. Since magnitude estimates in the present experiment were given once every $5 \mathrm{sec}$, the change in the magnitude estimate over $5 \mathrm{sec}$ that corresponded to the threshold of acceleration is $5 \times .0333$, or .165 . Therefore, a change in magnitude estimate of .165 or less over $5 \mathrm{sec}$ could be thought of as a point on the asymptote.

Based on the above argument, the point at which the asymptote was first reached could be obtained by comparing the values of consecutive pairs of $y$ on the fitted function. In the first instance, after the trial began, when the difference in magnitude between two consecutive $y_{s}$ values is less than or equal to .165 , asymptote is considered to be reached at the lower $x$ value. Differences in magnitudes between subsequent consecutive y pairs, according to the function, must be smaller than .165 .

(Manuscript received November 21, 1980; revision accepted for publication May 22, 1981.) 